\title{
Demonstration of $2 \mathrm{~mm}$ thick microcontrolled injectable stimulators based on rectification of high frequency current bursts
}

\author{
Laura Becerra-Fajardo, Student Member, IEEE, Marieluise Schmidbauer, Antoni Ivorra
}

\begin{abstract}
Existing implantable stimulators use powering approaches that result in stiff and bulky systems or result in systems incapable of producing the current magnitudes required for neuromuscular stimulation. This hampers their use in neuroprostheses for paralysis. We previously demonstrated an electrical stimulation method based on electronic rectification of high frequency (HF) current bursts. The implants act as rectifiers of $\mathrm{HF}$ current that flows through the tissues by galvanic coupling, transforming this current into low frequency current capable of performing neuromuscular stimulation. Here we developed $2 \mathbf{~ m m}$ thick, semi-rigid, injectable and addressable stimulators made of off-the-shelf components and based on this method. The devices were tested in vitro to illustrate how they are powered by galvanic coupling. In addition they were tried in an animal model to demonstrate their ability to perform controlled electrical stimulation. The implants were deployed by injection into two antagonist muscles of an anesthetized rabbit and were addressed resulting in independent isometric contractions. Low frequency currents of $2 \mathrm{~mA}$ were delivered by the implants. The HF currents are safe in terms of unwanted electrostimulation and tissue heating according to standards. This indicates that the proposed electrical stimulation method will allow unprecedented levels of miniaturization for neuroprostheses.
\end{abstract}

Index Terms- Galvanic coupling, microstimulator, neuroprostheses, rectifiers.

\section{INTRODUCTION}

$\mathrm{M}$ OTOR neuroprostheses elicit neuromuscular activity by performing electrical stimulation of peripheral nerves for restoring the function of impaired nervous systems [1]. Implantable neuroprosthetic systems are largely preferred over superficial and percutaneous configurations in long-term clinical applications mainly because superficial systems lack selectivity while percutaneous systems are less safe and comfortable [2]. However, implantable systems can be too invasive in some scenarios. In this regard, the need for an

Manuscript received April 6, 2016; revised August 1, 2016; accepted October 27, 2016.

L. Becerra-Fajardo is with the Department of Information and Communication Technologies, Universitat Pompeu Fabra, Barcelona, Spain (e-mail: laura.becerra.fajardo@gmail.com).

M. Schmidbauer was with the Department of Electrical and Computer Engineering, Technische Universitat Munchen, Germany, (e-mail: luiseschmidbauer@web.de).

A. Ivorra is a Serra Húnter Fellow, Department of Information and Communication Technologies, Universitat Pompeu Fabra, Barcelona, Spain (e-mail: antoni.ivorra@upf.edu). adequate and reliable power supply can represent a critical limitation for implantable neuroprostheses [3], especially in neuromuscular stimulators, which demand miniaturization and power in the order of the $\mathrm{mW}$. These two features partially drive their expansion in clinical applications [4].

Batteries and inductive coupling have been widely used for implantable wireless devices. However, both powering approaches exhibit some significant disadvantages. Low energy density of existing batteries makes them inadequate for power demanding applications in which miniaturization is a must [5]. In the case of inductive coupling, a number of factors hinder its use, including coupling misalignment [6] and the size of the receiving coil located in the implantable device. In the field of neuromuscular stimulation implants, BION microstimulators can be regarded as state-of-the-art case studies for both approaches with versions powered only by inductive coupling $(\varnothing=2 \mathrm{~mm})$ and versions powered by a rechargeable battery $(\varnothing=3.3 \mathrm{~mm})$ [7]. Another significant disadvantage of conventional inductive coupling is its incapability to power deep seated implants. This limitation has been partially addressed by Poon et al. by confining electromagnetic energy transport through propagating modes in tissue (mid-field) [6]. Yet, the solution by Poon et al. does not avoid the need for an implant coil with a diameter above $1 \mathrm{~mm}$ as theorized by Heetderks for neuromuscular stimulation [8]. The minimum coil diameter of the implants demonstrated by Poon et al. is $1.6 \mathrm{~mm}$ [9]. Other powering methods have been explored, including power transfer by near-infrared light [10], [11] and energy harvesters (e.g. piezoelectric [12] and natural processes of the body such as electric potentials of the inner ear [13]). However, these approaches do not accomplish sufficient power for neuromuscular stimulation, and/or are too invasive to be easily deployed in tissues [3], [14].

As an alternative to the above approaches, in [15] we proposed an electrical stimulation method based on the use of implants that perform electronic rectification of innocuous high frequency (HF) current bursts. These auxiliary HF bursts are supplied by skin electrodes and flow through the tissues where the implants are located by means of galvanic coupling. Each implant picks up a minute portion of this HF current to power up an electronic circuit that controls the flow of rectified, low frequency (LF) current capable of performing stimulation of local excitable tissues. These implants lack 
internal batteries or coils; they consist only of electronic components. Therefore, they can be implemented in a single integrated circuit or hybrid microcircuit connected to two electrodes at opposite ends of a flexible body. The proposed method requires a minimum voltage drop across the implant electrodes for operation. This implies that a minimum distance must be kept between them (from some millimeters to a very few centimeters for neuromuscular stimulation). For example, in the case of stimulation of the forearm muscles, we showed in [16] that a minimum distance of $3 \mathrm{~cm}$ is needed between the implant electrodes to use auxiliary HF currents with magnitudes that meet international safety standards.

We have already in vivo demonstrated non-addressable, flexible injectable devices $(\varnothing=1 \mathrm{~mm})$ which consist of a few passive components and which are capable of performing charge-balanced local electrical stimulation [17]. However, these simple stimulators lacked a mechanism to independently control them. This feature is required if multiple devices are to be deployed to perform complex stimulation patterns for movement restoration.

Here we report the development and evaluation of microcontrolled injectable stimulators which operate by rectifying bursts of HF current. The implants are made only of commercially available components mounted on a rigidflexible printed circuit board (rigid-flex PCB). Their elongated body is capable of picking up enough HF current to power up their electronics and deliver currents capable of neuromuscular stimulation, and they are sufficiently thin to be deployed by injection $(\varnothing=2 \mathrm{~mm})$. They can be considered as low-cost intermediate proof-of-concept prototypes towards ultrathin $(\varnothing$ $<1 \mathrm{~mm}$ ) addressable implants based on application-specific integrated circuits (ASICs). To the best of our knowledge, the assayed implants are not only the first injectable and controllable devices based on off-the-shelf components but are also the first injectable devices powered only by galvanic coupling.

\section{METHODS}

\section{A. Electrical stimulation system}

(The circuit architecture and operation of the microcontrolled stimulator was detailed in [18].)

The implantable stimulators consist in a narrow, elongated and semi-rigid tubular implant (Fig. $1 \mathrm{~A}$ ). The cylindrical structure houses an electronic circuit based only on off-theshelf components. Fig. $1 \mathrm{C}$ shows a simplified diagram of the circuit architecture. This architecture was detailed and demonstrated in [18]. Briefly, the implant electrodes pick up a portion of the HF current for powering the circuitry through a bridge rectifier. A regulator subcircuit stabilizes the supplied voltage to the control unit (CU). The amplitude demodulator compares two signals that have been low-pass filtered from the full-wave rectified signal to extract information from the same HF current. The demodulated signal is sent to the CU, which drives two current sources that generate LF biphasic symmetric waveforms for stimulation. These current sources act as peak current limiters for the rectified current that flows

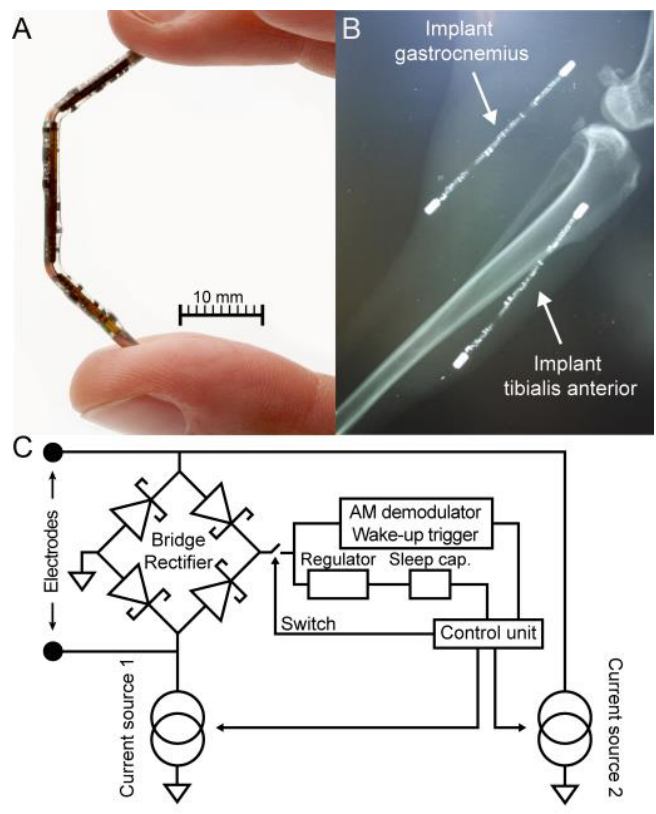

Fig. 1. Microcontrolled injectable stimulator $(\varnothing=2 \mathrm{~mm})$. A) The off-theshelf components are soldered on a rigid-flexible PCB, and are enclosed in a silicone tube with two stainless steel electrodes at opposite ends. B) X-ray images of the stimulators implanted in the tibialis anterior and gastrocnemius muscles. C) Basic circuit architecture of the injectable stimulator (see Methods section).

through the implant. In effect, LF current pulses are generated through the implant. During these current pulses, a switch is opened to guarantee that the LF current flows through the tissues, and not through the implant's subcircuits. The CU sleeps in-between stimulating bursts to reduce power consumption. When a burst is detected by the circuit, a wakeup trigger is generated and the $\mathrm{CU}$ activates to perform stimulation. A capacitor ('sleep capacitor' in Fig. 1 C) electrically feeds the wake-up trigger circuit and the $\mathrm{CU}$ inbetween bursts, avoiding the reset of the CU in every HF burst delivered by the external system.

The $\mathrm{CU}$ of the implantable stimulator is based on what nowadays seems to be the smallest commercially available programmable device: the $1.555 \times 1.403 \mathrm{~mm}$ ATtiny 20 (ATTINY20-UUR by Atmel Corp.). It is an 8 bit, low-power microcontroller with software selectable power saving modes, critical to ensure that the device remains in a low-energy consumption mode in-between HF bursts. The Schottky diodes used in the external prototypes described in [18] were replaced with diodes that had a lower forward voltage (NSR02F30NXT5G by ON Semiconductor). As a result, the $\mathrm{CU}$ inside the stimulator activated with a lower voltage drop across the implant electrodes, improving the system's performance.

The standard electronic components were assembled by soldering on the top and bottom layers of a $1.5 \times 45 \mathrm{~mm}$ dynamic rigid-flex PCB manufactured under the IPC-6013 Type 4 specification (L. A. B. Circuits S. A.). This PCB was made up of 6 layers consisting of 2 sets of 2 layers of copper on a rigid substrate, separated by 2 layers of copper placed over a flexible polyimide. To ensure flexibility, the PCB was 
divided in three rigid sections (average length of $13.7 \mathrm{~mm}$ ) divided by two flexible sections (length $=2 \mathrm{~mm}$ ).

The assembly process for the injectable stimulators can be summarized as follows. 1) Two components with ball grid array (BGA) packaging were soldered in an oven using the solder reflow temperature profile indicated by Atmel Corp. for the microcontroller. 2) The rest of the components were soldered using a soldering iron, tweezers and a microscope. 3) The assembled circuit was gently inserted in a platinum cured silicone tube (721048 by Harvard Apparatus) that has a $1.9 \mathrm{~mm}$ outside diameter. 4) The tube was filled with a biocompatible silicone (MED-6015 by NuSil Technology) which has low viscosity when uncured. Each end of the PCB included a copper pad for the electrode assembly. After curing the silicone, the excess of silicone on the pads was removed to guarantee good electrical contact. 5) Using conductive epoxy (CW2400 by Chemtronics), the two copper pads were cold soldered to the inner end wall of $2 \mathrm{~mm}$ diameter stainless steel closed-end tubes cut to a length of $3.8 \mathrm{~mm}$ (Beijing ShengRuiKe Automation Equipment Co. Ltd). 6) Any empty spaces left by the epoxy in the pad-electrode union were filled with silicone to protect the epoxy from body fluids. The $48.7 \mathrm{~mm}$ long implantable stimulator has a diameter of $2 \mathrm{~mm}$ and a mass of $0.4 \mathrm{~g}$.

The external generator (Fig. $2 \mathrm{~A}$ ), including the hardware and the communication protocol, was described in depth in [18]. A computer running a LabVIEW virtual instrument (National Instruments Corp.) generates a modulating signal using a data acquisition (DAQ) board (NI-USB6216 by National Instruments Corp.). A function generator (AFG3022 by Tektronix, Inc.) uses this signal to modulate a $1 \mathrm{MHz}$ sinusoidal voltage carrier by means of amplitude-shift keying (ASK). The modulated signal is then amplified using a high voltage amplifier (WMA 300 by Falco Systems) and delivered across the target tissue using a pair of electrodes. For the in vitro assays, these electrodes consisted of aluminum plates. In the case of the in vivo experimentation, the external electrodes consisted of $3 \mathrm{~cm}$ wide bands made from silver-based stretchable conductive fabric (MedTex P-180 by Statex) strapped around the rabbit's hind limb, as shown in Fig. 4 A.

The communication protocol is based on the scheme described in [18]. ASK is used to send data modulated on the HF auxiliary current $(f=1 \mathrm{MHz})$. The modulating signal generated by the LabVIEW virtual instrument uses Manchester coding, which offers two important advantages: 1) has no dc component, as required by the external system to avoid charge injection with the HF auxiliary current and 2) a clock signal can be recovered from the encoded data. This self-clocking feature allows that, during the decoding process, the $\mathrm{CU}$ resynchronizes in every bit.

The transmitted signal consists of three distinguishable stages that have specific relative amplitudes. The first stage is an $85 \mathrm{~ms}$ low amplitude unmodulated signal used as Power up. This stage initially powers and stabilizes the circuit and it is particularly long because of the power up sequence of the microcontroller. The second stage consists in a modulated Synch\&Data signal used for synchronization and for transmission of data. This stage has a sequence of 3 synchronizing rising-edge transitions, and a 9 bit data stream ( 8 address bits followed by 1 parity bit). Bits are sent at a baud rate of $25 \mathrm{kBd}$. After this stage, a $200 \mu \mathrm{s}$ zero-amplitude slot is used for processing (decoding, parity bit check and address validation to perform stimulation). The third stage consists in an unmodulated signal of maximum amplitude for the Stimulation bursts. This stage is able to wake up the microcontroller and perform the biphasic symmetric waveform for stimulation (e.g. $200 \mu \mathrm{s}+30 \mu \mathrm{s}+200 \mu \mathrm{s}$ ).

\section{B. Safety analysis}

To illustrate that the delivered HF current bursts can be safe in terms of unwanted electrostimulation and tissue heating, it was calculated the root mean square (rms) value of the applied electric field $\left(\mid \mathbf{E}_{\mathrm{rms}}\right)$ and the specific absorption rate (SAR) for the above stimulation protocol and variations of it plausible in neuromuscular stimulation scenarios. The rms value can be expressed as:

$$
\left|\mathbf{E}_{\text {rms }}\right|=\sqrt[2]{\frac{\int_{0}^{t} \mathbf{E}_{\text {peak }}{ }^{2} d t}{t}}
$$

Assuming that in a time $t$ the external system only initializes the implants once (Power up stage), sends information for one address (Synch\&Data) and uses the remaining time to transmit stimulation bursts, the $\left|\mathbf{E}_{\text {rms }}\right|$ could be expressed as:

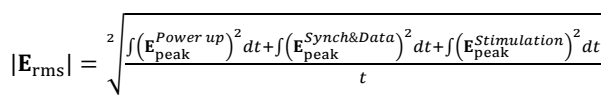

The Power up stage has a relative amplitude $k_{1}$ with respect to the applied field $\left|\mathbf{E}_{\text {peak }}\right|$. The Synch\&Data stage has a relative amplitude $k_{2}$. Having these values in mind, (2) could be rewritten as:

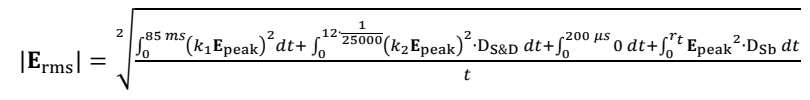

Where $r_{t}$ is the remaining time to apply stimulation bursts, $\mathrm{D}_{\mathrm{S} \& \mathrm{D}}$ is the duty cycle during Synch\&Data (equal to 0.5 due to Manchester encoding), and $\mathrm{D}_{\mathrm{Sb}}$ is the duty cycle during the stimulation phase, and is defined as:

$$
\mathrm{D}_{\mathrm{Sb}}=\mathrm{F} \cdot\left(\text { pulse } e_{\text {width }} \cdot 2+50 \mu \mathrm{s}\right)
$$

Where $\mathrm{F}$ is the stimulation frequency. As explained in [18], $k_{1}$ and $k_{2}$ are defined respectively as 0.65 and 0.75 , as the former relative amplitude is capable of initializing the implantable circuitry, while the latter compensates the discharge of the capacitors during the low levels of the Synch\&Data stage and the processing slot. The HF external generator is capable of defining both the frequency of stimulation and the pulse width of the biphasic symmetric current waveform before generating the HF current bursts. Then, the rms value of the electric field will depend in both stimulation parameters.

In terms of tissue heating due to the HF current, IEEE standard specifies a maximum threshold defined as the 
Specific Absorption Rate (SAR) [19]. It can be related to the electric field at a point by the expression:

$$
\mathrm{SAR}=\frac{\sigma}{\rho}\left|\mathbf{E}_{\mathrm{rms}}\right|^{2} \quad \mathrm{~W} / \mathrm{kg}
$$

Where $\sigma$ is the tissue electrical conductivity $(\mathrm{S} / \mathrm{m}), \rho$ is the tissue mass density $\left(\mathrm{kg} / \mathrm{m}^{3}\right)$, and $\left|\mathbf{E}_{\mathrm{rms}}\right|$ is the applied electric field rms magnitude $(\mathrm{V} / \mathrm{m})$. A muscle density of $1000 \mathrm{~kg} / \mathrm{m}^{3}$ [20] and conductivity of $0.5 \mathrm{~S} / \mathrm{m}$ at $1 \mathrm{MHz}$ [21] are assumed.

\section{Measurement setups}

The implant picks up a portion of the HF current and rectifies it internally to generate a dc voltage for powering its circuitry. This implies that a minimum voltage drop must be obtained across the implant electrodes for operation. Alignment between the implant axis and the HF electric field would accomplish the maximum voltage gradient between electrodes. However, this ideal scenario may not be possible for two reasons: 1) physical implantation constraints and 2) relative rotation of the implant with respect to the external electrodes during normal operation.

To evaluate this, it was implemented an in vitro experimental setup (Fig. 2 A) where the implants were placed at different angles with respect to the electric field. Two
$7 \mathrm{~cm} \times 2.5 \mathrm{~cm}$ aluminum plates acting as external electrodes $(1.2 \mathrm{~mm}$ thick, 1050A) and connected to the external generator, were held parallel at a distance of $11 \mathrm{~cm}$ using two polycarbonate plates. A $1 \mathrm{~cm} \times 1 \mathrm{~cm}$ grid made of cotton thread was sewed across the plates, $1.25 \mathrm{~cm}$ from the bottom of the structure. The structure was placed inside a $19 \mathrm{~cm} \times$ $14 \mathrm{~cm} \times 6.3 \mathrm{~cm}$ glass container filled up to $3 \mathrm{~cm}$ with saline $(0.33 \% \mathrm{NaCl})$ with a conductivity of $0.51 \mathrm{~S} / \mathrm{m}$ which approximately corresponds to the admittivity magnitude of skeletal muscle at $1 \mathrm{MHz}$ [21].

Four implants were laid on the grid and were wirelessly activated by the external generator using the saline as the galvanic coupling medium. Multiple trials were performed in which the angle of the implants with respect to the plates and the distance between implants was varied. Each implant was programmed with its own address. In this in vitro study, in order to facilitate recognition of the implant activation, one current source inside the injectable device was replaced with a LED before the encapsulation process. Two thin wires connected to an oscilloscope (TPS2014 by Tektronix, Inc.) were placed in contact to the implant electrodes to record the voltage drop across a "dummy implant" that does not draw any current and a real implant that powers up but is not addressed by the external generator. Voltages were recorded
A
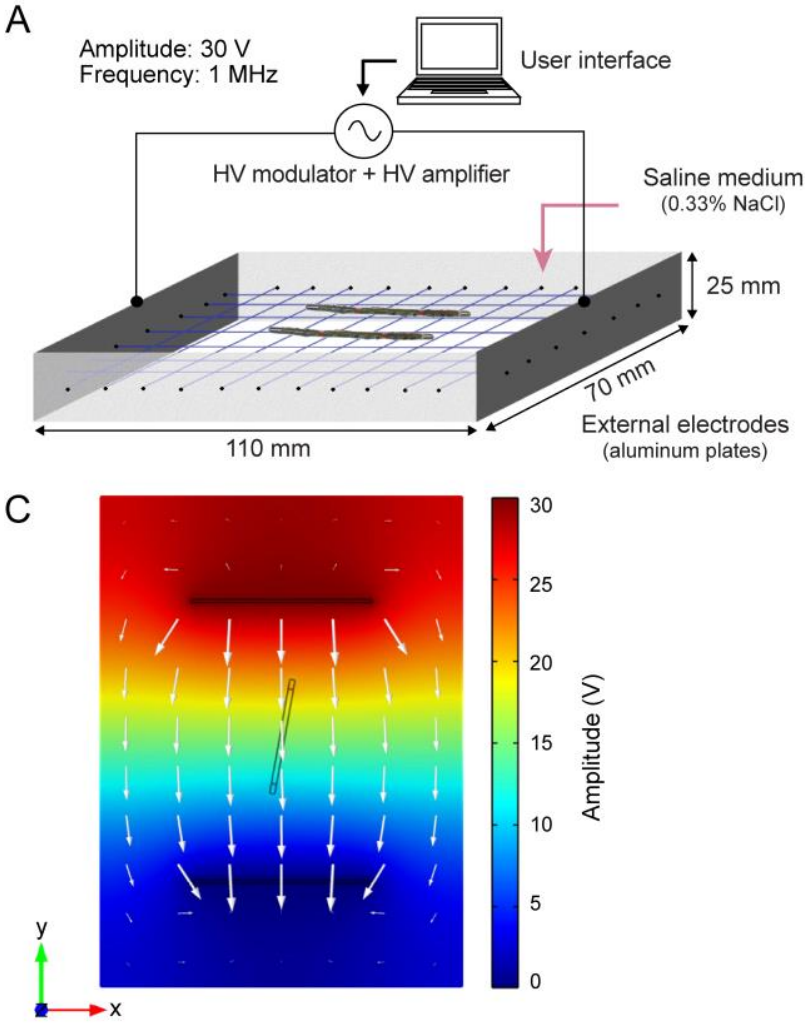

B
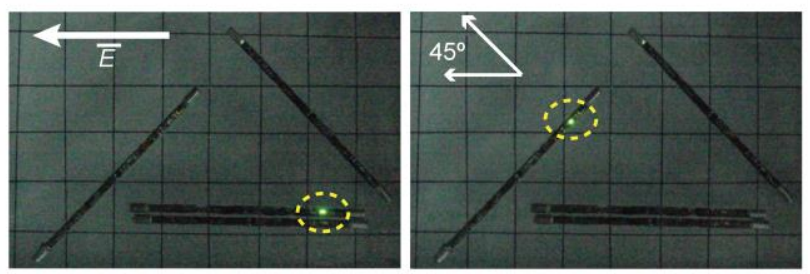

D

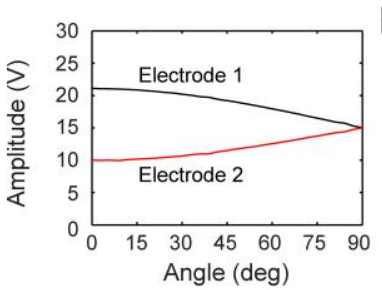

E

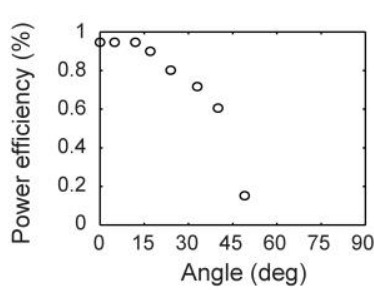

$\mathrm{F}$

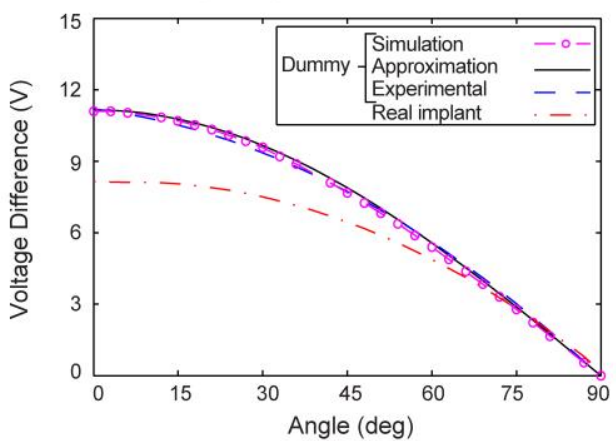

Fig. 2. In vitro demonstration and simulation. A) Two aluminum external electrodes are held parallel and separated by two polycarbonate plates. The structure supports a regular grid made of cotton thread and is immersed into a glass container filled with saline. Four implants are placed over the grid. A user interface commands the modulation of a sinusoidal $1 \mathrm{MHz}$ carrier, which is amplified and delivered through the external electrodes. B) Pictures of $\mathrm{n}$ addressed stimulators $(\mathrm{n}=4)$. The implants activation is manifested by lighting LEDs (noted with dashed ovals). Even when the implants are placed close together, they independently operate (left). The implants can be powered and activated even if they are tilted $45^{\circ}$ with respect to the applied field (right). C) FEM simulation result of the amplitude of the ac electric potential (color scale) and of the electric field relative magnitude and direction (white arrows). It is simulated the delivery of an ac voltage $(\mathrm{A}=30 \mathrm{~V})$ across the external electrodes. A dummy implant is modeled at the center of the setup, and a parametric analysis is performed to evaluate the electric potential at the implant electrodes depending on its tilt. D) Simulation results for the dummy implant electrodes. E) Power efficiency of the system during stimulation $(\mathrm{F}=100 \mathrm{~Hz})$. F) Comparison of the electric potential difference obtained for the dummy implant in the FEM simulation, the approximation of (6) and the in vitro recordings, with the real implant voltage drop recorded in saline. 
for different tilts.

To calculate the efficiency of the system, the power delivered by the implant during stimulation was measured by recording the LF voltage across the implant electrodes during a Stimulation burst. To do so, two thin wires connected to a RC low-pass filter with high input impedance (cut-off frequency $=7.2 \mathrm{kHz}$ ) were placed in contact to the implant electrodes of a real implant. The device was addressed by the external system, and the LF voltage during stimulation was recorded using an oscilloscope (TPS2014 by Tektronix, Inc.).

The in vitro setup was analyzed by means of a Finite Element Method (FEM) software platform (COMSOL Multiphysics) using the 'Electric Currents' application mode to perform a parametric study of electric potential at the electrodes of a dummy implant. The angle $\theta$ between the implant axis and the electric field was increased from $0^{\circ}$ to $90^{\circ}$ at $3^{\circ}$ steps. The modeled dummy implant consists of an insulating tube (length $=41 \mathrm{~mm}$, diameter $=2 \mathrm{~mm}$, conductivity $=1 \times 10^{-4} \mathrm{~S} / \mathrm{m}$, relative permittivity $=11.7$ ) with two metallic cylinders at opposite ends (length $=3.8 \mathrm{~mm}$, diameter $=2 \mathrm{~mm}$, conductivity $=1 \times 10^{4} \mathrm{~S} / \mathrm{m}$, relative permittivity $=1$ ). The saline medium is modeled with a conductivity of $0.5 \mathrm{~S} / \mathrm{m}$ and a relative permittivity of 1836.4 [21]. The software automatically generated a mesh of 7464 tetrahedral elements. The implant electrodes were defined as probes, and the electric potential calculated during the parametric simulation was exported to a numerical computing software (Matlab, by Mathworks, Inc) for further graphical analysis.

Before deploying a stimulator in the tibialis anterior (TA) muscle of an anesthetized rabbit, the operation of the injectable device was demonstrated externally by connecting it to a percutaneous bipolar probe which was deployed at the motor point of the muscle where the stimulator would be later implanted. The probe consisted of a $1.8 \mathrm{~mm}$ diameter coaxial cable (50CX-41 by Temp-Flex Cable Inc.) whose core and shield conductor were contacted to two $3.8 \mathrm{~mm}$ long, $2 \mathrm{~mm}$ thick stainless steel electrodes separated by a $41 \mathrm{~mm}$ long fluorinated ethylene propylene (FEP) insulator. These dimensions are similar to those of the implantable stimulators. The distal electrode acts as the stimulation electrode whereas the proximal one acts as the return electrode. The probe was connected in series to the implant and to the parallel combination of a $10 \Omega$ resistor and a $2.2 \mu \mathrm{F}$ capacitor (lowpass filter LPF, cut-off frequency $=7.2 \mathrm{kHz}$ ) as shown in Fig. 3 A. This setup allows recording the voltage drop across the implant electrodes and the LF components of the electric current flowing through the device using an oscilloscope (TPS2014 by Tektronix, Inc.).

After the implants' deployment, isometric plantarflexion and dorsiflexion forces were recorded using a load cell (STC - 10kgAL-S by Vishay Precision Group, Inc.) mounted on a custom-made acrylic board (Fig. 4 A). The animal was lying sideways, the hock was fixed to the horizontal surface using an atraumatic padded clamp and the foot was tied with clamps to the load cell. The force signal was recorded with a DAQ at a rate of $100 \mathrm{kHz}$ using a signal conditioning electronic circuit that included a first order LPF with a cutoff frequency of $500 \mathrm{~Hz}$.

\section{Animal Handling}

The animal procedure was approved by the Ethical Committee for Animal Research of the Barcelona Biomedical Research Park (CEEA-PRBB), application number: JMC 141606. One New Zealand White male rabbit weighting $4.46 \mathrm{~kg}$ was employed. For sedation and initial anesthesia, Dexmedetomidine $(0.9 \mathrm{~mL})$, Butorfanol $(0.45 \mathrm{~mL})$ and Ketamine $(0.45 \mathrm{~mL})$ were intramuscularly administered between 15 to 30 minutes prior to the preparation of the animal. Then, the right hind limb of the rabbit was shaved, from the head of the femur to the mid tarsus. In addition, a depilatory cream (Veet sensitive skin, by Reckitt Benckiser
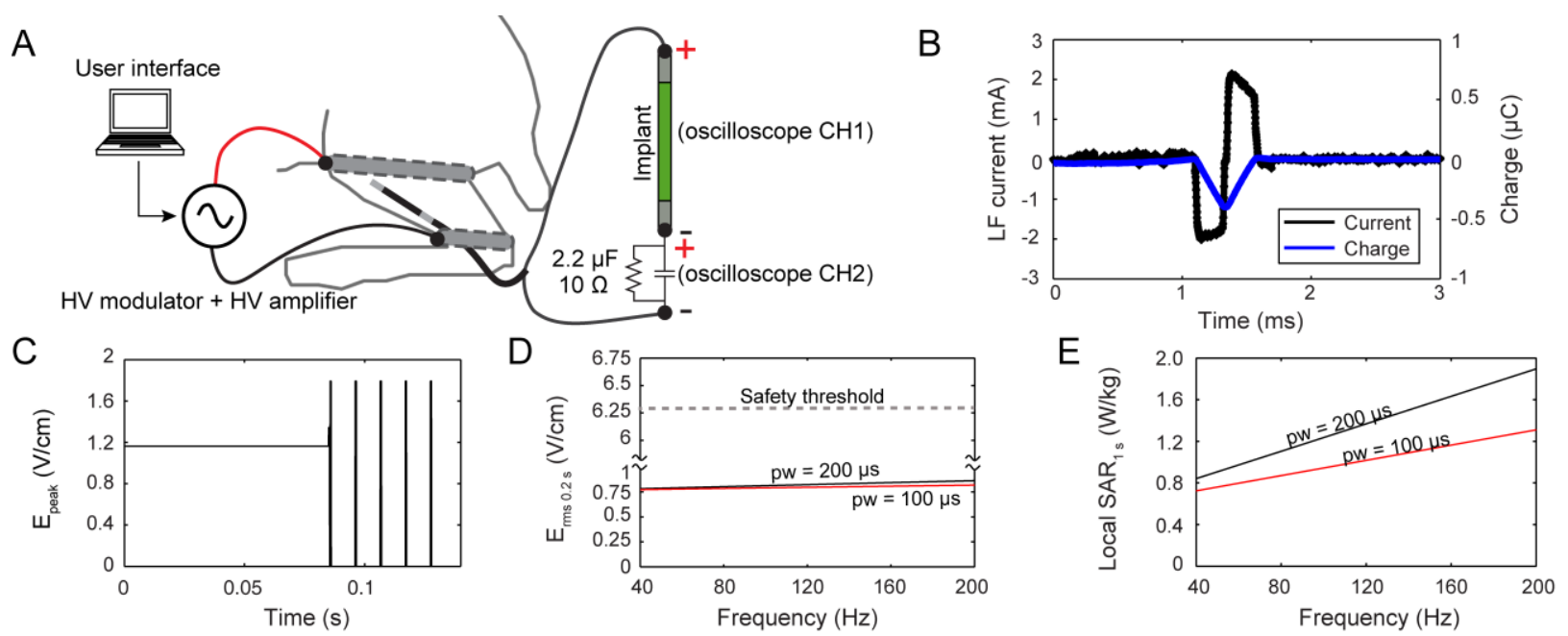

Fig. 3. In vivo experiments: electric measurement. A) Setup using a bipolar probe implanted in the tibialis anterior (TA) muscle and connected to the implant and a RC low-pass filter. B) LF current delivered by the implant in the TA and the calculated charge injection. A dc-blocking capacitor balanced any charge mismatch occurring during the delivery of the biphasic symmetric current pulses. C) Estimated HF electric field magnitude at the location of the bipolar probe (and later the location of the implant) during the three stages of the HF signal (Power up, Synch\&Data, Stimulation bursts). D) $\left|\mathrm{E}_{\mathrm{rms}}\right|$ averaged over $0.2 \mathrm{~s}$ for different stimulation pulse widths and frequencies, and the safety threshold. E) Local SAR averaged over $1 \mathrm{~s}$ for different stimulation pulse widths and frequencies. 
Group plc) was applied for 5 min and thoroughly rinsed. The animal was transferred to an anesthetic circuit using endotracheal intubation and anesthesia was maintained with Isoflurane (1-1.5\%). Ringer's lactate $(11 \mathrm{~mL} / \mathrm{h})$ was administered intravenously, and the animal was constantly monitored with a capnograph and pulse oximeter. A heating pad was employed during the entire session. At the end of the study, the animal was euthanized with an overdose of Thiobarbital sodium (8 mL IV).

\section{E. Implantation procedure}

The implantation procedure, inspired by the deployment system and sequence described in [7], is illustrated in Fig. 5. At first, the target muscle was identified by palpation and the approximate site for deploying the implant (i.e. motor point) was located using anatomical cues. It was assumed that such motor point was close to the origin of the muscle. A $2.1 \mathrm{~mm}$ thick catheter (14 G, model 382268 by Becton, Dickinson and Company) was longitudinally introduced from the hock up to this end, resulting in an insertion depth of about $6 \mathrm{~cm}$. The motor point of the muscle was verified using the tip of the catheter's needle as an exploration electrode and an $\mathrm{Ag} / \mathrm{AgCl}$ gel electrode (model 2228 by $3 \mathrm{M}$ ) placed on the thigh of the animal as a return electrode. A custom-made current generator delivered through the electrodes 1 to $6 \mathrm{~mA}$ symmetric biphasic pulses (pulse width $=200 \mu \mathrm{s}$ ) at $100 \mathrm{~Hz}$. If the induced movement was deemed too weak or did not match the expected joint movement, the catheter was repositioned by inserting or extracting the catheter a few millimeters.

After the motor point was identified, the insertion point of the catheter was marked to avoid dislocation, and the needle was extracted. Then, the proximal end of the catheter was cut, and a $3.4 \mathrm{~mm}$ catheter $(10 \mathrm{G}$, model 382287 by Becton, Dickinson and Company) used as dilator was gently slid down the $14 \mathrm{G}$ catheter until a mark in the dilator matched the marked insertion point, indicating that both catheter tips were lined up. The $14 \mathrm{G}$ catheter was gently extracted from the dilator (Fig. 5 B), and a mark was placed on the dilator and skin to avoid dislocation.

At first, LF currents and voltage drop across the implant electrodes were measured using the bipolar probe described above. The probe was inserted in the dilator until a mark on the probe lined up with the proximal end of the dilator, indicating that the tip of the probe was located by the identified motor point. The dilator was gently extracted, until the two electrodes of the probe were in contact with the tissues, and the external measurements were performed. Then, the dilator was gently inserted back into the tissues, the marks were lined up assuring that its tip was in the motor point, and the probe was extracted.

A surgical thread was tied to the anode of the implantable stimulator in case the stimulator had to be withdrawn after deployment. Then, the stimulator was introduced into the dilator (Fig. 5 C), with the cathode facing the dilator's tip, and it was pushed towards the identified motor point using the custom-made probe (Fig. $5 \mathrm{D}$ ). When a specific mark on the probe matched the proximal end of the dilator (assuring that
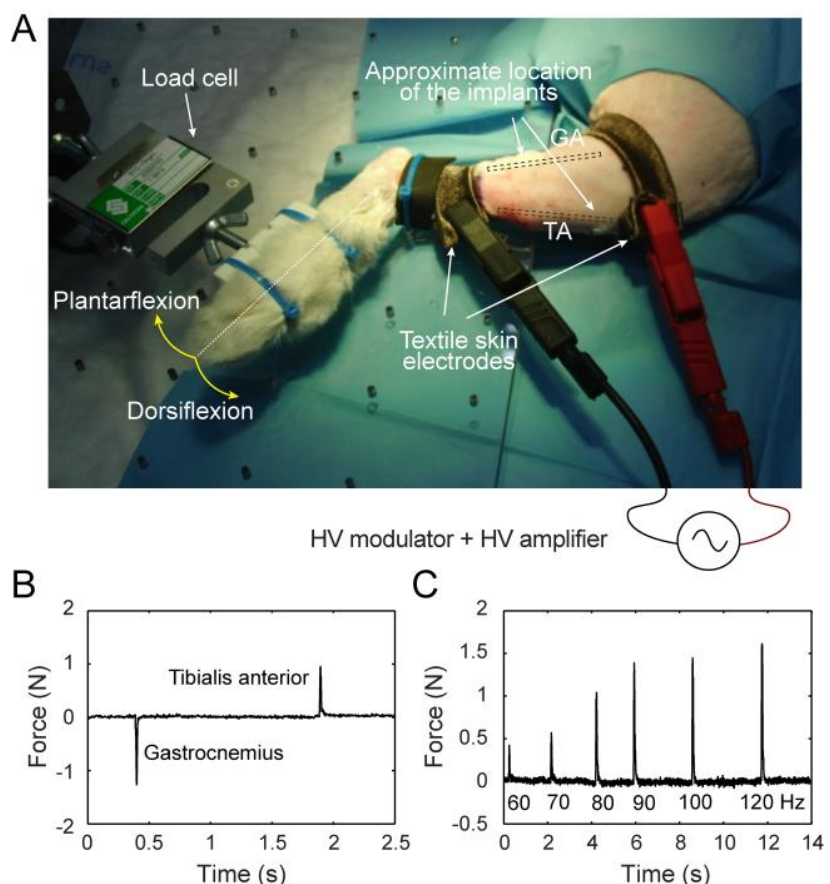

Fig. 4. In vivo experiments: isometric force measurements. A) Setup with implants deployed by injection into the TA and gastrocnemius (GA) muscles. The hock of the animal was fixed to a horizontal surface and the foot was fixed to a load cell to measure dorsiflexion and plantarflexion twitch contractions. The textile electrodes were strapped around the hind limb and were connected to the external system. B) Force recordings. The user independently addressed one or the other implant to induce plantarflexion or dorsiflexion movements, and C) defined the frequency of stimulation to modulate the force magnitude.

the implant's cathode was in contact with the motor point), the dilator was cautiously withdrawn by holding firmly the probe, releasing the implant inside the muscle. Finally, the probe was removed, and the insertion point was treated (Fig. 5 E). No bleeding was visible. The implantation procedure was carried out both in the TA and the gastrocnemius (GA) muscles of the right hind limb of the rabbit.

\section{RESULTS}

The developed injectable stimulator (length $=48.7 \mathrm{~mm} ; \varnothing=$ $2 \mathrm{~mm}$ ) is composed of a semi-rigid PCB enclosed in a silicone tubing with two stainless steel electrodes at opposite ends (Fig. 1 A). Its small diameter allows deployment using a catheter, and its length guarantees a sufficient voltage drop to electrically feed its electronic circuit and generate the LF currents required for stimulation.

Four implants were placed in the in vitro setup shown in Fig. 2 A. They were powered and independently addressed using a HF bursts generator $(f=1 \mathrm{MHz}, \mathrm{A}=30 \mathrm{~V}$, distance between electrodes $=11 \mathrm{~cm}$ ). The HF electric field magnitude in this scenario was $2.72 \mathrm{~V} / \mathrm{cm}$. Two still frames are shown in Fig. $2 \mathrm{~B}$. The frame on the left shows the activation of an implant aligned to the electric field applied by the external generator. Even though this implant is separated only $1 \mathrm{~mm}$ from another implant, the system is capable of powering and independently addressing that implant. The frame on the right shows the activation of an implant with a $45^{\circ}$ angle with respect to the electric field. In this case, as reported in the 
simulations presented below, the amplitude of the ac voltage drop across the implant electrodes was expected to be about 7.7 $\mathrm{V}$ under the assumption that the implant does not draw any current (i.e. dummy implant). In reality, the voltage drop was $6.4 \mathrm{~V}$ as measured by contacting thin wires to the implant electrodes. This is enough to power the $\mathrm{CU}$ inside the injectable device and turn on the LED that shows the implant's activation. At larger angles the devices cannot be powered with this electric field magnitude. These results show that the injectable stimulators can activate even if they are not perfectly aligned to the delivered electric field.

The FEM simulation results are shown in Fig. 2 C, D and F. Fig. $2 \mathrm{C}$ shows the amplitude of the ac electric potential generated by the external system and the corresponding electric field when the implant was tilted $10^{\circ}$. Fig. $2 \mathrm{D}$ shows the electric potential amplitude measured at the electrodes of the dummy implant. The maximum voltage drop amplitude across the implant electrodes is $11.1 \mathrm{~V}$ when the implant is perfectly aligned to the electric field $\left(0^{\circ}\right)$. For a tilt of $45^{\circ}$ the voltage drop is $7.7 \mathrm{~V}$. These simulations indicate that, despite the implant electrodes are relatively large (length $=3.8 \mathrm{~mm}$ $\varnothing$ $=2 \mathrm{~mm}$ ) the amplitude of the voltage drop across the implant electrodes can be approximated by the expression:

$$
\Delta V=\cos \theta \cdot d \cdot\left|\mathbf{E}_{\text {peak }}\right|
$$

Where $\theta$ is the tilt angle, $d$ is the separation distance between the two implant electrodes, and $\left|\mathbf{E}_{\text {peak }}\right|$ is the peak electric field applied. Fig. $2 \mathrm{~F}$ shows the voltage drop obtained for the dummy implant in the FEM simulation, the approximation of (6) and the in vitro recordings; and that obtained with the real implant recorded in saline.

If the implants were to be used for continuous stimulation at $100 \mathrm{~Hz}$, and assuming that the system had to be initialized each second, the average HF power consumption of the external system would be $1.1 \mathrm{~W}$. FEM simulations show that at $100 \mathrm{~Hz}$, the impedance across the implant electrodes is $381 \Omega$. According to this impedance and the LF voltage across the implant electrodes recorded in vitro, the instantaneous power delivered by the implants ranges between 1.7 and $10.5 \mathrm{~mW}$ depending on the angle between the implant axis and the electric field. Fig. $2 \mathrm{E}$ shows the efficiency of the implant during stimulation $(\mathrm{F}=100 \mathrm{~Hz})$ for different tilts.

Electrical and force measurements were performed to test the implants in vivo. Fig. $3 \mathrm{~B}$ shows the recorded LF current and the charge injection computed from it. The implant was programmed to and was able to apply biphasic LF currents with a magnitude of $2 \mathrm{~mA}$ and a duration of $200 \mu \mathrm{s}+200 \mu \mathrm{s}$ with a dwell time of $30 \mu \mathrm{s}$. This implies that the implant's CU was able to decode the information sent through the HF signal and enable the current sources to generate a biphasic symmetric current waveform. If a charge mismatch appeared at the end of the anodic (positive) current pulse, the dcblocking capacitor of the implant passively balanced this charge in-between stimulating bursts. The bursts triggered a wake-up signal for the microcontroller to generate the
TABLE I

CALCUlated $\left|\mathbf{E}_{\text {RMS }}\right|(\mathrm{V} / \mathrm{m})$

\begin{tabular}{cccc}
\multicolumn{4}{c}{ CALCULATED $\mid \mathbf{E}_{\text {RMS }}(\mathrm{V} / \mathrm{m})$} \\
\hline \hline Pulse width $(\mu \mathrm{s})$ & $\mathrm{F}(\mathrm{Hz})$ & $\mathbf{E}_{\mathrm{rms}} 0.2 \mathrm{~s}(\mathrm{~V} / \mathrm{m})$ & $\mathbf{E}_{\mathrm{rms}} 1 \mathrm{~s}(\mathrm{~V} / \mathrm{m})$ \\
\hline 100 & 40 & 77.2 & 38.1 \\
& 80 & 78.4 & 41.7 \\
& 120 & 79.5 & 45.1 \\
& 160 & 80.7 & 48.2 \\
200 & 200 & 81.8 & 51.2 \\
& 40 & 78.1 & 41.0 \\
& 80 & 80.2 & 47.0 \\
& 120 & 82.3 & 52.3 \\
& 160 & 84.2 & 57.1 \\
& 200 & 86.2 & 61.6 \\
\hline \hline
\end{tabular}

stimulating waveforms, and the implant remained asleep between bursts, minimizing power consumption.

The amplitude of the $1 \mathrm{MHz}$ voltage applied across the external electrodes was $50 \mathrm{~V}$ during the Stimulation bursts. At these episodes, the voltage drop amplitude across the bipolar probe electrodes was $6.20 \mathrm{~V}$. According to the in vitro results and simulations reported above, this corresponds to a voltage drop of about $7.35 \mathrm{~V}$ under the assumption of a dummy implant and an estimated local field $\left(\left|\mathbf{E}_{\text {peak }}\right|\right)$ of about $1.79 \mathrm{~V} / \mathrm{cm}$. Fig. $3 \mathrm{C}$ shows the estimated peak electric field at the location of the implant during the three stages of the protocol delivered by the external HF generator (Power up, Synch\&Data, and Stimulation bursts) [18]. Both the generator and the implant were programmed so that the stimulation consisted of 5 biphasic waveforms at a frequency of $100 \mathrm{~Hz}$. For that the generator had to deliver 5 bursts of $450 \mu \mathrm{s}$.

Table 1 shows the calculated average $\left|\mathbf{E}_{\text {rms }}\right|$ during 0.2 and 1 s using stimulation frequencies ranging from 40 to $200 \mathrm{~Hz}$, and pulse widths of 100 and $200 \mu$ s using (3) and (4). These are typical stimulation frequencies that can be delivered by electrical stimulators (e.g. BION microstimulators [7]). A $0.2 \mathrm{~s}$ period is calculated as established by the IEEE to define the maximum in situ electric field for preventing unwanted electrostimulation [19]. The standard specifies that at $1 \mathrm{MHz}$ this $\left|\mathbf{E}_{\text {rms }}\right|$ must be lower than $6.26 \mathrm{~V} / \mathrm{cm}$ at the extremities. The maximum $\left|\mathbf{E}_{\mathrm{rms}}\right|$ obtained here is $0.86 \mathrm{~V} / \mathrm{cm}(f=200 \mathrm{~Hz}$, pulse width of $200 \mu$ s) (Fig. 3 D). Therefore, the HF bursts can be considered as safe in terms of unwanted electrostimulation. The same can be stated regarding SAR. Fig. $3 \mathrm{E}$ shows the local SAR obtained for each $\left|\mathbf{E}_{\text {rms }}\right|$ value averaged over $1 \mathrm{~s}$. IEEE safety standard specifies that the maximum localized SAR in controlled environments is $10 \mathrm{~W} / \mathrm{kg}$ averaged over $10 \mathrm{~g}$ of tissue [19]. In the worst case scenario analyzed here in which the external HF generator triggers a $200 \mathrm{~Hz}$ stimulation with a pulse width of $200 \mu$ s, the computed SAR is about $1.90 \mathrm{~W} / \mathrm{kg}$.

After the electrical measurements, the probe was extracted and the devices were deployed by injection into the target tissues using a $10 \mathrm{G}$ catheter. Stiffness was not appreciated by palpation of the animal's hind limb probably thanks to the implants semi-rigidity.

Fig. 4 shows the force measurement setup and the recordings obtained by independently activating the two implanted stimulators. The user was able to wirelessly address the implants and control the stimulation frequency. Plantarflexion and dorsiflexion twitch contractions were 
induced with magnitudes of 0.96 and $1.2 \mathrm{~N}$ respectively ( 5 bursts, $80 \mathrm{~Hz}$ ) as shown in Fig. $4 \mathrm{~B}$. The induced force could be modulated by varying the stimulation frequency (Fig. 4 C). A stimulation assay using the implanted devices is available as supplementary multimedia material.

After the stimulation assays, X-ray images were taken of the hind limb of the animal where the implants were deployed (Fig. $1 \mathrm{~B}$ ). The integrity of the implants was preserved during the whole study. After euthanasia, the implants were extracted, cleaned, inspected and electrically evaluated. It was verified that they were still fully functional in terms of addressability and stimulation capabilities.

\section{DISCUSSION}

The microcontrolled injectable stimulators described here are able to perform neuromuscular stimulation by rectifying epidermically applied HF current bursts.

We anticipate that the tubular, flexible and small conformation of the implants will mitigate issues faced during implantation procedures. Additionally, the injectable devices will be less sensitive to body movements [22], will help reduce the foreign body response [23] and will boosts their use in practical scenarios where minimal invasiveness is a must.

The in vivo experiment showed that these prototypes are easily injected into muscle tissue through the lumen of a catheter without causing bleeding. This same catheter can be employed to identify the target deployment site (motor point). Using a graphic interface that governed the modulation of the HF current bursts, the user defined which muscle to trigger and the repetition frequency of the bursts. This last parameter allowed gradual variation of the induced force, which is a common observation when conventional LF pulses are delivered for neuromuscular stimulation [24]. As stated in [17], this suggests that the half-rectified HF currents delivered by the implants are equivalent in terms of excitatory behavior to conventional LF current pulses.

There was no evidence that the HF current delivered by the external generator induced unwanted electrostimulation or tissue heating. Both the calculated $\left|\mathbf{E}_{\text {rms }}\right|$ and the local SAR comfortably comply with IEEE standards.

Galvanic coupling - which is the way the implants are energized here - has been extensively studied for intrabody communications [25]. However, the idea of using this approach as an energy source for implantable devices has not been explored thoroughly. This is confirmed in recent reviews on power approaches for implants in which galvanic coupling is not even mentioned [3], [14]. The reasons why other researchers were reluctant - or did not conceive - to use galvanic coupling for power can only be guessed. In our opinion, such reluctance mainly arises from not appreciating two facts. First, whereas moderate LF currents can be harmful, large HF currents can innocuously flow through the human body if applied as short bursts because they neither cause stimulation nor significant heating. Second, the implants can be shaped as thin and flexible elongated bodies for picking up a sufficient voltage drop. Such implant conformation is highly beneficial in terms of minimal invasiveness, not only because it allows percutaneous deployment but also because it minimizes tissue damage. That conformation is already massively used in clinical practice: thousands of women have been subdermally implanted with hormonal contraceptive implants which consist of a flexible rod that is about $4 \mathrm{~cm}$ long and $2 \mathrm{~mm}$ in diameter [26].

Galvanic coupling presents two drawbacks that were addressed in this study: 1) since the component of the electric field in the direction of the implant axis must be large enough for guaranteeing a sufficient voltage drop, some degree of alignment is required and 2) direct electrical contact between the external generator and the body tissues is necessary. The results show that even with a $45^{\circ}$ tilt, the implants can activate, thereby alignment is not a stringent requirement. We have also in vivo demonstrated that gel-free textile electrodes perform an adequate contact for delivering the HF currents that enable the implants to operate. This is possible due to the low contact impedance that textile electrodes exhibit at high frequencies [27]. They could be easily embedded in garments for creating comfortable external systems.

Even though galvanic coupling presents very low power efficiency, we have demonstrated in vitro that the power required by the external system to operate the implants for continuous stimulation could be delivered using existing rechargeable portable batteries (> $100 \mathrm{Wh} / \mathrm{kg}$ ). To corroborate
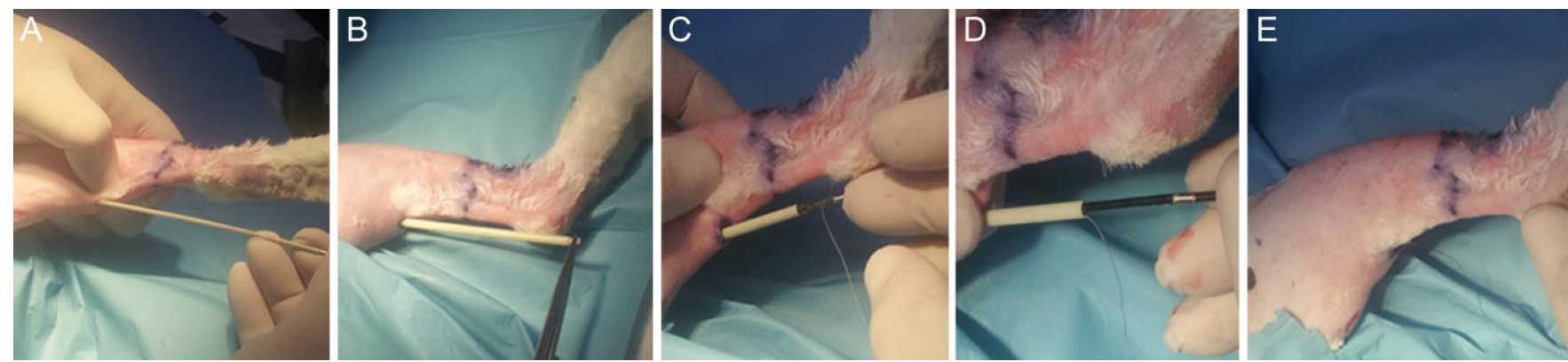

Fig. 5. Implantation procedure. A) The approximate site for deploying the implant was located by anatomical cues. A $14 \mathrm{G}$ catheter was introduced towards the muscle's origin. The tip of the catheter's needle was used as an exploration electrode to locate the point of maximum excitability (i.e. motor point). For that, an electro-stimulator was connected across the catheter needle and an external return electrode and the insertion depth of the catheter was adjusted until a strong contraction was noticed. B) The needle was extracted from the catheter, and a $10 \mathrm{G}$ catheter was inserted over the $14 \mathrm{G}$ catheter to dilate the orifice. The $14 \mathrm{G}$ catheter was extracted. C-D) The implant was inserted through the $10 \mathrm{G}$ catheter and pushed towards the identified motor point. E) The dilator was extracted and the external return electrode was detached. The surgical thread observed in C-E was tied to the implant to pull it out in case it was necessary to remove it for replacement. 
this in a model that resembles more closely a real neuromuscular stimulation scenario, we have performed numerical analyses (not reported) with realistic anatomical computer models [28]. The results show that power consumption for using these implants in the human limbs will range from about $1 \mathrm{~W}$ to $10 \mathrm{~W}$ depending on the considered stimulation application (e.g. target muscle, stimulation frequency and duration). This confirms that those power requirements are attainable in realistic medical devices by using existing rechargeable portable batteries.

The demonstrated implants consist only of off-the-shelf components mounted on a rigid-flex PCB. This use of low cost technologies suggests that these devices could be widely accessible for experimental studies. Aiming at further miniaturization, multiple strategies could be explored to improve the implants' design. The width of the PCB was mainly constrained by the size of the biggest component: the microcontroller. As electronic component manufacturers tend to offer further miniaturization, we anticipate that smaller similar implants will be feasible in the near future. This can also be applicable to other relatively large electronic components within the design. Furthermore, the implants' biggest passive component, the dc-blocking capacitor, could be avoided using other strategies to prevent dc currents such as the charge counter we demonstrated in [29]. The manufacturing capabilities of the PCB provider also constrained the implants' length. Aspects such as the minimum diameter of the annular ring in the internal layers $(190 \mu \mathrm{m})$ and the minimum distance between conductors $(125 \mu \mathrm{m})$ are likely to improve in the near future. As explained above, the demonstrated implants are considered as proof-ofconcept prototypes towards ultrathin addressable implants based on ASICs. These ultrathin microstimulators will not have these important limitations, as all the electronic components will be integrated into a single circuit with a length of a very few millimeters.

The demonstrated systems are only capable of electrical stimulation and do not possess sensing capabilities. In the near future, we plan to embed detection and quantification of electromyographic signals which could be picked up with the implant electrodes. These sensing capabilities could, for instance, allow the implementation of artificial proprioception for closed-loop control [30]. We have already in vitro demonstrated bidirectional communications based on this electrical stimulation method [31].

\section{CONCLUSIONS}

Electronic rectification of $\mathrm{HF}$ current bursts allows the development of addressable neuromuscular stimulators which can be composed only of commercial components and which can be shaped as semi-rigid thin devices suitable for implantation by injection. In particular, the addressable implants reported here, composed of a relatively long $(\sim 5 \mathrm{~cm})$ semi-rigid circuit enclosed in a silicone tube $(\varnothing=2 \mathrm{~mm})$, were easily deployed into the target tissues through a $10 \mathrm{G}$ catheter. Their operation was demonstrated in the hind limb of an anesthetized rabbit, accomplishing controlled and independent joint movements.

The HF currents that power the implants circuitry and that are transformed by them into LF currents capable of local stimulation, are innocuous according to safety standards because they do not cause unwanted stimulation, due to their $\mathrm{HF}$, and because they do not cause significant heating, due to the fact that they are applied in short bursts. These auxiliary bursts can be delivered through textile skin electrodes without any need for gels or skin preparations aimed at improving the electrical contact. Alignment of the implants with the generated HF fields is not a stringent requirement. In here it was demonstrated that even with a $45^{\circ}$ tilt between the implant's axis and the electric field, the devices were powered and responded to activation requests by the external system.

By demonstrating an alternative to existing methods for power transfer/generation such as inductive coupling or batteries, these results pave the way to the development of sophisticated electronic implants unprecedented in terms of minimal invasiveness.

\section{ACKNOWLEDGMENT}

The authors express their gratitude to J.M. Caballero and S. Capdevila (PRBB animal facility) for their assistance in writing the animal protocol, and to $\mathrm{J}$. Grífols (Zoològic Badalona Veterinària) for his work regarding all animal procedures. LBF's research is supported by a PRC fellowship from the UPF. AI's research was supported by a Marie Curie grant (IRG 256376) from the European Commission.

\section{REFERENCES}

[1] J. T. Mortimer and N. Bhadra, "Chapter 11 - Fundamentals of Electrical Stimulation," in Neuromodulation, P. H. P. Elliot S. Krames A. Hunter Peckham and Ali R. Rezai Elliot S. Krames and A. R. Rezai, Eds. San Diego: Academic Press, 2009, pp. 109-121.

[2] P. H. Peckham and J. S. Knutson, "Functional Electrical Stimulation for Neuromuscular Applications," Апnи. Rev. Biomed. Eng., vol. 7, no. 1, pp. 327-360, 2005.

[3] A. Kim, M. Ochoa, R. Rahimi, and B. Ziaie, "New and Emerging Energy Sources for Implantable Wireless Microdevices," IEEE Access, vol. 3, pp. 89-98, 2015.

[4] V. Pikov, "Global market for implanted neuroprostheses," Implant. Neuroprostheses Restoring Funct., p. 383, 2015.

[5] M. Rasouli and L. S. J. Phee, "Energy sources and their development for application in medical devices.," Expert Rev. Med. Devices, vol. 7, no. 5, pp. 693-709, Sep. 2010.

[6] J. S. Ho, A. J. Yeh, E. Neofytou, S. Kim, Y. Tanabe, B. Patlolla, R. E. Beygui, and A. S. Y. Poon, "Wireless power transfer to deep-tissue microimplants," Proc. Natl. Acad. Sci., pp. 7974-7979, May 2014.

[7] J. H. Schulman, "The Feasible FES System: Battery Powered BION Stimulator," Proc. IEEE, vol. 96, no. 7, pp. 1226-1239, 2008.

[8] W. J. Heetderks, "RF powering of millimeter- and submillimeter-sized neural prosthetic implants.," IEEE Trans. Biomed. Eng., vol. 35, no. 5, pp. 323-7, May 1988.

[9] K. L. Montgomery, A. J. Yeh, J. S. Ho, V. Tsao, S. Mohan Iyer, L. Grosenick, E. A. Ferenczi, Y. Tanabe, K. Deisseroth, S. L. Delp, and A. S. Y. Poon, "Wirelessly powered, fully internal optogenetics for brain, spinal and peripheral circuits in mice," Nat Meth, vol. 12, no. 10, pp. 969-974, Oct. 2015.

[10] A. Abdo, M. Sahin, D. S. Freedman, E. Cevik, P. S. Spuhler, and M. S. Unlu, "Floating light-activated microelectrical stimulators tested in the rat spinal cord.," J. Neural Eng., vol. 8, no. 5, p. 056012, Oct. 2011.

[11] H. Liu, T. Zhao, W. Jiang, R. Jia, D. Niu, G. Qiu, L. Fan, X. Li, W. Liu, B. Chen, Y. Shi, L. Yin, and B. Lu, "Flexible Battery-Less Bioelectronic 
Implants: Wireless Powering and Manipulation by Near-Infrared Light," Adv. Funct. Mater., vol. 25, no. 45, pp. 7071-7079, Dec. 2015.

[12] C. Dagdeviren, B. D. Yang, Y. Su, P. L. Tran, P. Joe, E. Anderson, J. Xia, V. Doraiswamy, B. Dehdashti, X. Feng, B. Lu, R. Poston, Z. Khalpey, R. Ghaffari, Y. Huang, M. J. Slepian, and J. A. Rogers, "Conformal piezoelectric energy harvesting and storage from motions of the heart, lung, and diaphragm.," Proc. Natl. Acad. Sci. U. S. A., vol. 111, no. 5, pp. 1927-32, Feb. 2014.

[13] P. P. Mercier, A. C. Lysaght, S. Bandyopadhyay, A. P. Chandrakasan, and K. M. Stankovic, "Energy extraction from the biologic battery in the inner ear.," Nat. Biotechnol., vol. 30, no. 12, pp. 1240-3, Dec. 2012.

[14] A. Ben Amar, A. B. Kouki, and H. Cao, "Power Approaches for Implantable Medical Devices.," Sensors, vol. 15, no. 11, pp. 28889-914, 2015.

[15] A. Ivorra, "Remote Electrical Stimulation by Means of Implanted Rectifiers," PLoS One, vol. 6, no. 8, p. e23456, 2011.

[16] A. Ivorra and L. Becerra-Fajardo, "Wireless Microstimulators Based on Electronic Rectification of Epidermically Applied Currents: Safety and Portability Analysis," in 18th IFESS Annual Conference, 2013, pp. $213-$ 216.

[17] A. Ivorra, L. Becerra-Fajardo, and Q. Castellví, "In vivo demonstration of injectable microstimulators based on charge-balanced rectification of epidermically applied currents," J. Neural Eng., vol. 12, no. 6, p. 66010, 2015.

[18] L. Becerra-Fajardo and A. Ivorra, "In Vivo Demonstration of Addressable Microstimulators Powered by Rectification of Epidermically Applied Currents for Miniaturized Neuroprostheses," PLoS One, vol. 10, no. 7, p. e0131666, Jul. 2015.

[19] "IEEE Standard for Safety Levels with Respect to Human Exposure to Radio Frequency Electromagnetic Fields, $3 \mathrm{kHz}$ to $300 \mathrm{GHz}$," pp. 1238, Apr. 2006.

[20] A. Ibrahiem, C. Dale, W. Tabbara, and J. Wiart, "Analysis of the Temperature Increase Linked to the Power Induced by RF Source," Prog. Electromagn. Res., vol. 52, pp. 23-46, 2005.

[21] S. Gabriel, R. W. Lau, and C. Gabriel, "The dielectric properties of biological tissues: III. Parametric models for the dielectric spectrum of tissues," Phys. Med. Biol., vol. 41, no. 11, pp. 2271-2293, Nov. 1996.

[22] E. Salman and M. Stanacevic, "3-D Integrated Implantable Device for Deep Brain Sensing and Stimulation," CEWIT-WWHI Joint Symposium on Wireless Medicine and Health Technologies, 2011. [Online]. Available: http://www.ece.sunysb.edu/ milutin/pubs/2011/cewit2011_3d.pdf.

[23] J. L. Skousen, S. M. E. Merriam, O. Srivannavit, G. Perlin, K. D. Wise, and P. A. Tresco, "Reducing surface area while maintaining implant penetrating profile lowers the brain foreign body response to chronically implanted planar silicon microelectrode arrays.," Prog. Brain Res., vol. 194, pp. 167-80, Jan. 2011.

[24] T. Kesar, L.-W. Chou, and S. A. Binder-Macleod, "Effects of stimulation frequency versus pulse duration modulation on muscle fatigue.," J. Electromyogr. Kinesiol., vol. 18, no. 4, pp. 662-71, Aug. 2008.

[25] M. Seyedi, B. Kibret, D. T. H. Lai, and M. Faulkner, "A survey on intrabody communications for body area network applications.," Biomed. Eng. IEEE Trans., vol. 60, no. 8, pp. 2067-79, Aug. 2013.

[26] P. D. Blumenthal, K. Gemzell-Danielsson, and M. Marintcheva-Petrova, "Tolerability and clinical safety of Implanon.," Eur. J. Contracept. Reprod. Health Care, vol. 13 Suppl 1, pp. 29-36, Jun. 2008

[27] L. Beckmann, C. Neuhaus, G. Medrano, N. Jungbecker, M. Walter, T. Gries, and S. Leonhardt, "Characterization of textile electrodes and conductors using standardized measurement setups.," Physiol. Meas., vol. 31, no. 2, pp. 233-47, Feb. 2010.

[28] A. Christ, W. Kainz, E. G. Hahn, K. Honegger, M. Zefferer, E. Neufeld, W. Rascher, R. Janka, W. Bautz, J. Chen, B. Kiefer, P. Schmitt, H.-P. Hollenbach, J. Shen, M. Oberle, D. Szczerba, A. Kam, J. W. Guag, and N. Kuster, "The Virtual Family-development of surface-based anatomical models of two adults and two children for dosimetric simulations," Phys. Med. Biol., vol. 55, no. 2, pp. N23-N38, Jan. 2010.

[29] L. Becerra-Fajardo and A. Ivorra, "Charge Counter for Performing Active Charge-Balance in Miniaturized Electrical Stimulators," in 6th European Conference of the International Federation for Medical and Biological Engineering SE - 64, vol. 45, 2015, pp. 256-259.

[30] D. R. Merrill, J. Lockhart, P. R. Troyk, R. F. Weir, and D. L. Hankin, "Development of an implantable myoelectric sensor for advanced prosthesis control.," Artif. Organs, vol. 35, no. 3, pp. 249-52, Mar. 2011.

[31] L. Becerra-Fajardo and A. Ivorra, "Bidirectional communications in wireless microstimulators based on electronic rectification of epidermically applied currents," in Neural Engineering (NER), 2015 7th International IEEE/EMBS Conference on, 2015, pp. 545-548.

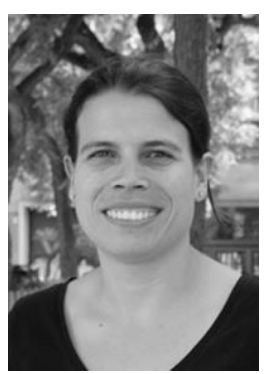

Laura Becerra-Fajardo received the B.Sc. degree in electronic engineering from Universidad Nacional de Colombia in 2008, the M.Sc. degree in biomedical engineering from Universitat de Barcelona and Universitat Politècnica de Catalunya in 2012 and the Ph.D. degree from the Department of Information and Communication Technologies of Universitat Pompeu Fabra, Barcelona, in 2016. Her research focuses mainly in exploring and designing medical devices capable of performing electrical stimulation for neuroprostheses.

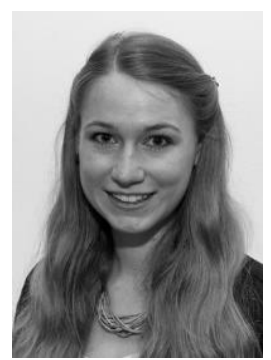

Marieluise Schmidbauer received the B.Sc. degree in electrical engineering in 2013, and the M.Sc. degree in electrical engineering in 2016, both from the Technical University in Munich. During her studies she worked at the HeinzNixdorf Chair for Medical Electronics. In 2015 she was an exchange student at the Universitat de Pompeu Fabra, working and completing her master thesis in the Biomedical Electronics Research Group (BERG). Since 2016 she is working for InnoME GmbH.

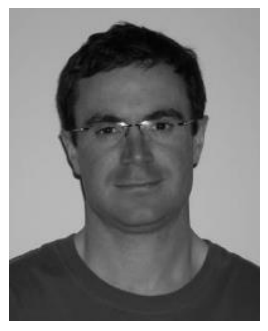

Antoni Ivorra received a Ph.D. degree in electronics engineering from the Universitat Politècnica de Catalunya in 2005. Between 1998 and 2005, he was with the Biomedical Applications Group of the Centre Nacional de Microelectrònica, Spain. He enjoyed a three-year postdoctoral position at the University of California at Berkeley (2005-2008) followed by an appointment as Assistant Research Engineer at the same institution for a year and a second postdoctoral position at the CNRS - Institut Gustave Roussy, France, for eight months.

Dr. Ivorra is currently an Associate Professor at the Universitat Pompeu Fabra. His main research topics are electroporation, electrical bioimpedance and neuroprosthetics. 